\title{
Solución Numérica de Ecuación de Burgers
}

\section{Juan Luna ${ }^{1}$}

(Recibido: 19/08/2016

\section{Edith Carhuapoma ${ }^{2}$}

Aceptado: 05/10/2016)

Resumen: En este trabajo se estudia el problema de Burgers en el caso simple, se indican algunos resultados de existencia, unicidad y regularidad de la solución del problema, y en su forma variacional se da un resultado que da una condición suficiente para que una función sea solución débil. Pero como la solución puede presentar discontinuidades, entonces pasaremos a una nueva ecuación agregando un término de viscosidad artificial, obteniendo así una ecuación viscosa cuya solución, por un resultado, converge a la solución de la ecuación de Burgers cuando el término viscoso tiende a cero. Por tanto estudiaremos la ecuación viscosa desde el punto de vista numérico.

Palabras Claves: Esquemas de discretización, Vicosidad artificial, Problema variacional, Operador de proyección, Matriz de precondicionamiento.

\section{Numerical Solution of Burgers Equation}

Abstract: In this paper the problem of Burgers is studied in the simplest case, some results of existence, uniqueness and regularity of the solution of the problem are indicated, and in its variational form it is given a result that gives a sufficient condition for a function to be weak solution. But as the solution may present discontinuities, then we pass on to a new equation by adding a term of artificial viscosity, thus obtaining a viscous solution for equation whose results converge to the solution of the Burgers equation when the viscous term tends to zero. Therefore we study the viscous equation from a numerical point of view.

Key Words: Discretization schemes, artificial Vicosidad, Variational problem, Projection operator. Preconditioning matrix.

\section{Introducción}

Los problemas físicos están determinados en general por ecuaciones en derivadas parciales no lineales. Obtener la solución analítica muchas veces no es posible, por tanto es necesario determinar la solución numérica y para ello se estudia los algoritmos que nos permiten obtener dicha solución numérica evitando mayores dificultades. Primero discretizaremos en la variable temporal, luego se aproximan en las variables espaciales. Utilizaremos el método de mínimos cuadrados y el algoritmo del gradiente conjugado, la cual nos permite utilizar matrices simétrica que en cada etapa no cambia, y además no es necesario que el dato inicial esté cerca de la solución.

La ecuación de Burgers en la forma unidimensional es la ecuación de Euler para los fluidos ideales

$$
\left\{\begin{array}{c}
\frac{\partial u}{\partial t}+u \frac{\partial u}{\partial x}=0, \quad x \in R, \quad t>0 \\
u(x, 0)=u_{0}(x)
\end{array}\right.
$$

\footnotetext{
${ }^{1}$ UNMSM, Facultad de Ciencias Matemáticas, e-mail: jlunav@unmsm.edu.pe

${ }^{2}$ UNMSM, Facultad de Ciencias Matemáticas, e-mail: milagross302ecl@hotmail.com
} 
La ecuación (i) es un caso particular de una ecuación hiperbólica no lineal que está dado por

$$
\frac{\partial u}{\partial t}+\frac{\partial u}{\partial x}[f(u)]=0
$$

La solución de la ecuación (i) puede admitir discontinuidades, por tanto, al discretizar el problema correspondiente, se tiene que entre los puntos de la malla más cercanos a la discontinuidad, existe una variación de $u$ que no tiende a cero. Así, en esta zona no se puede plantear un esquema de diferencias finitas. Para evitar ésta dificultad se perturba la ecuación de Burgers agregando el término $-\gamma \frac{\partial^{2} u}{\partial x^{2}}$, denominado viscosidad artificial, con el coeficiente $\gamma>0$ y pequeño.

Por tanto, la ecuación viscosa de Burger esta dado por

$$
\frac{\partial u}{\partial t}+u \frac{\partial u}{\partial x}-\gamma \frac{\partial^{2} u}{\partial x^{2}}=0
$$

Esta ecuación es lo que se va estudiar.

\section{Metodología}

\subsection{Formulación del problema}

Consideremos el siguiente problema no lineal

$$
\left\{\begin{array}{c}
\frac{\partial u}{\partial t}+\frac{\partial}{\partial x}[f(u)]-\gamma \frac{\partial^{2} u}{\partial x^{2}}=0 \quad(x, t) \in \mathbb{R} \times(0,+\infty) \\
u(x, 0)=u_{0}(x) \quad x \in \mathbb{R}
\end{array}\right.
$$

es un problema viscoso, donde $f, u_{0}: \mathbb{R} \rightarrow \mathbb{R}$ son funciones dadas.

Para resolver este problema se discretizará primero con respecto a la variable $t$, generando así una sucesión de problemas estacionarios. Considerando el siguiente esquema para la discretización en tiempo $\Delta t>0$ se tiene

$$
t_{n}=n \Delta t \quad y \quad u^{n}(x) \simeq u(x, n \Delta t)
$$

de tal manera que

$$
\frac{u^{n+1}-u^{n}}{\Delta t}+\frac{d}{d x}\left[f\left(u^{n+1}\right)\right]-\gamma \frac{d^{2} u}{d x^{2}}=0
$$

como $u_{0}$ es de soporte compacto, entonces tomando un $R$ adecuado, la condición inicial puede reescribirse como

$$
u^{n+1}(-R)=u^{n+1}(+R)=0
$$

Luego, la semidescritización respecto al tiempo en (1) se obtiene el problema estacionario para $u^{n+1}$

$$
\left\{\begin{array}{c}
\frac{u^{n+1}-u^{n}}{\Delta t}+\frac{d}{d x}\left[f\left(u^{n+1}\right)\right]-\gamma \frac{d^{2} u}{d x^{2}}=0 \\
u^{n+1}(-R)=u^{n+1}(+R) \stackrel{=0}{=}
\end{array}\right.
$$

éste problema puede transformarse, mediante un cambio de variables sobre el intervalo [0,1], en

$$
\left\{\begin{array}{c}
\frac{u^{n+1}}{\Delta t}+\frac{d}{d x}\left[f\left(u^{n+1}\right)\right]-\gamma \frac{d^{2} u}{d x^{2}}=\frac{u^{n}}{\Delta t} \\
u^{n+1}(0)=u^{n+1}(1) \stackrel{=}{=}
\end{array}\right.
$$

Estudiaremos la ecuación de Burgers viscosa y estacionaria siguiente

$$
\left\{\begin{array}{c}
\alpha u+\frac{1}{2} \frac{d}{d x}\left(u^{2}\right)-\gamma \frac{d^{2} u}{d x^{2}}=f \\
u(0)=u(1)=0
\end{array}\right.
$$


donde $f \in L^{2}(0,1)$ o $\left(f \in H^{-1}(0,1)\right)$.

La ecuación (1) se obtiene a partir de la ecuación (4), haciendo $\alpha=1, f(u)=\frac{1}{2} u^{2}$.

\subsection{Formulación variacional}

En la ecuación (4) multiplicamos por funciones test $v \in D(0,1)$ e integrando por partes, se tiene

$$
\alpha \int_{0}^{1} u v d x+\int_{0}^{1} u \frac{d u}{d x} v d x+\gamma \int_{0}^{1} \frac{d u}{d x} \frac{d v}{d x} d x=\int_{0}^{1} f v d x
$$

por densidad de $D(0,1)$ en $H_{0}^{1}(0,1)$, se obtiene la formulación variacional de (1):

Hallar $u \in H_{0}^{1}(0,1)$ tal que

$$
\left\{\begin{array}{c}
\alpha \int_{0}^{1} u v d x+\int_{0}^{1} u \frac{d u}{d x} v d x+\gamma \int_{0}^{1} \frac{d u}{d x} \frac{d v}{d x} d x=\int_{0}^{1} f v d x \\
\forall v \in H_{0}^{1}(0,1)
\end{array}\right.
$$

Los términos de esta ecuación estan bien definidos, veamos:

1. Si $f \in H^{-1}(0,1)$ entonces se obtiene el mismo problema con $\langle f, v\rangle_{H^{-1}, H_{0}^{1}}$ en el segundo miembro de la ecuación.

2. Dado que $u, v \in H_{0}^{1}(0,1)$ se tiene

$$
u, v \in L^{2}(0,1) \quad y \quad \frac{d u}{d x}, \frac{d v}{d x} \in L^{2}(0,1)
$$

Además se tiene, por resultados en los espacios de Sobolev sobre inyección, que

$$
H_{0}^{1}(0,1) \hookrightarrow L^{4}(0,1) \Rightarrow u, v \in L^{4}(0,1)
$$

Así, podremos aplicar la desigualdad de Hölder a las integrales que aparecen en (5)

$$
\begin{aligned}
\int_{0}^{1} u v d x & \leq\|u\|_{L^{2}}\|v\|_{L^{2}} \\
\int_{0}^{1} u \frac{d u}{d x} v d x & \leq\|u\|_{L^{4}}\left\|\frac{d u}{d x}\right\|_{L^{2}}\|v\|_{L^{4}} \leq C\|u\|_{H_{0}^{1}}\|v\|_{L^{4}} \\
\int_{0}^{1} \frac{d u}{d x} \frac{d v}{d x} d x & \leq\left\|\frac{d u}{d x}\right\|_{L^{2}}\left\|\frac{d v}{d x}\right\|_{L^{2}} \leq C\|u\|_{H_{0}^{1}}\|v\|_{H_{0}^{1}} \\
\int_{0}^{1} f v d x & \leq\|f\|_{L^{2}}\|v\|_{L^{2}}
\end{aligned}
$$

\subsection{Existencia de solución del problema de Burgers variacional}

En el problema (5) la dificultad se da por ser un problema no lineal. La existencia del problema está basada en la técnica del punto fijo. Haremos uso de algunos resultados.

Teorema 2.1. (Schauder) Sean $X$ un espacio de Banach, $M \subset X$ un convexo cerrado y $M \neq \phi$, $F: X \longmapsto X$ operador compacto tal que $F(M) \subset M$.

Entonces

$$
\exists x \in M \quad \text { tal que } F(x)=x
$$

Demostración. Ver [1] , [3]

Teorema 2.2. El problema (5) admite solución $u \in H_{0}^{1}$

Demostración. Ver [1], [3] 


\subsection{Solución numérica del problema de Burgers estacionario}

\section{Formulación del problema aproximado}

Se va utilizar el método de elementos finitos para obtener un problema en dimensión finita que sea una aproximación del problema variacional (5).

Sean $N>0, h=\frac{1}{N+1}, x_{i}=i h, \quad 0 \leq i \leq N+1$

Consideremos la aproximación interna de $H_{0}^{1}(0,1)$ dada por el subespacio $V_{0 h}$ definida por

$$
V_{0 h}=\left\{v_{h} \in C^{0}([0,1]) / v_{h}(0)=v_{h}(1)=0 ; \text { para } v_{h} \in\left[x_{i}, x_{i+1}\right], \quad i=0,1, \ldots, N\right\}
$$

El conjunto de funciones:

$$
B_{0 h}=\left\{w_{i}\right\}_{i}^{N} \subset V_{0 h} \quad \text { con } w_{i}\left(x_{j}\right)=\delta_{i j} \quad i, j \in\{1,2, \ldots, N\}
$$

forman una base del espacio $V_{0 h}$.

Aplicando el método de Galerkin, se tiene el problema aproximado:

Hallar $u_{h} \in V_{0 h}$ tal que

$$
\alpha \int_{0}^{1} u_{h} v_{h} d x+\int_{0}^{1} u_{h} \frac{d u_{h}}{d x} v_{h} d x+\gamma \int_{0}^{1} \frac{d u_{h}}{d x} \frac{d v_{h}}{d x} d x=\int_{0}^{1} f_{h} v_{h} d x, \forall v_{h} \in V_{0 h}
$$

Definición 2.3. Se define el operador de proyección $r_{h}$ como

$$
\begin{gathered}
r_{h}: H_{0}^{1}(0,1) \longmapsto V_{0 h} \\
v \longrightarrow r_{h}(v)
\end{gathered}
$$

con

$$
r_{h}(v)\left(x_{i}\right)=v\left(x_{i}\right), \quad \forall i=1,2, \ldots, N
$$

Definición 2.4. Sea $\Omega \subset R^{N}$ un abierto, se dice que la familia $\left\{\Omega_{r}\right\}_{r=1}^{M}$ es una partición de $\Omega$ si

(i) $\Omega_{r} \subset \Omega$ es abierto, $\forall r=1,2, \ldots, M$

(ii) $\Omega_{r} \cap \Omega_{s}=\phi, \quad \forall r, s=1,2, \ldots, M, \quad r \neq s$

(iii) $\Omega=\operatorname{int}\left(\cup_{r=1}^{M} \bar{\Omega}_{r}\right)$

Teorema 2.5. Sea $\Omega \subset R^{N}$ un abierto acotado. Para cada $M \geq 1$, la partición $\left\{\Omega_{r}\right\}_{r=1}^{M}$ de $\Omega$, con $m\left(\Omega_{i}\right)=\int_{\Omega_{i}} d x>0, d x=d x_{1} \ldots d x_{n}$. Sean $f \in L^{p}(\Omega), \quad 1 \leq p<\infty$ y $f_{M}=$ $\sum_{i=1}^{M}\left[\frac{\int_{\Omega_{i}} d x}{m\left(\Omega_{i}\right)} \chi_{\Omega_{i}}\right]$. Supongamos que $\lim _{M \rightarrow \infty}\left[\sup _{i}\left(\operatorname{diam}\left(\Omega_{i}\right)\right)\right]=0$.

Entonces

$$
\lim _{M \rightarrow \infty} f_{M}=f \quad \text { en } \quad L^{p}(\Omega)
$$

Demostración. Ver [1], [3].

Teorema 2.6. Sea el operador

$$
r_{h}: H_{0}^{1}(0,1) \longmapsto H_{0}^{1}(0,1)
$$

una bola cerrada $W \subset H_{0}^{1}(0,1)$ tal que $r_{h}(W) \subset W$ entonces

$$
\exists v \in W \text { tal que } \lim _{h \rightarrow 0}\left\|r_{h}(v)-v\right\|_{H_{0}^{1}}=0
$$


Demostración. Será suficiente demostrar que

$$
\lim _{h \rightarrow 0}\left\|\frac{d}{d x} r_{h}(v)-\frac{d v}{d x}\right\|_{L^{2}}=0
$$

Sea $e_{i}=\left[x_{i-1}, x_{i}\right]$, entonces

$$
\begin{aligned}
\frac{d}{d x} r_{h}(v) & =\sum_{i=1}^{N+1}\left[\frac{r_{h}(v)\left(x_{i}\right)-r_{h}(v)\left(x_{i-1}\right)}{h} \chi_{e_{i}}\right]=\sum_{i=1}^{N+1}\left[\frac{v\left(x_{i}\right)-v\left(x_{i-1}\right)}{h} \chi_{e_{i}}\right] \\
& =\sum_{i=1}^{N+1}\left[\frac{\int_{x_{i-1}}^{x_{i}} v^{\prime}(\xi) d \xi}{m\left(e_{i}\right)} \chi_{e_{i}}\right]=\sum_{i=1}^{N+1}\left[\frac{\int_{e_{i}} v^{\prime}(\xi) d \xi}{m\left(e_{i}\right)} \chi_{e_{i}}\right]
\end{aligned}
$$

por el teorema anterior, se tiene

$$
\frac{d}{d x} r_{h}(v) \longrightarrow v^{\prime} \text { en } L^{2}(0,1)
$$

con lo cual

$$
r_{h}(v) \longrightarrow v \text { en } H_{0}^{1}(0,1)
$$

Para mayor detalle, ver [1], [3].

\subsection{Solución del problema aproximado}

El siguiente resultado asegura la existencia de la solución del problema aproximado.

Teorema 2.7. El problema (7) admite al menos una solución.

Demostración. Ver [3], [4]

Teorema 2.8. (Brower) Sea $X$ un espacio de dimensión finita, $T$ un operador continuo de $X$ en si mismo y $B \subset X$ un convexo, cerrado y acotado tal que $T(B) \subset B$. Entonces

$$
\exists x \in B \text { tal que } T(x)=x
$$

la elección particular

$T=T_{h}$

$B=B_{h}=\left\{v \in V_{0 h} \quad /\|v\|_{H_{0}^{1}} \leq \frac{C}{\gamma}\|f\|_{L^{2}}\right\}$

esto permite concluir la existencia de, al menos, una solución $u_{h} \in V_{0 h}$.

Demostración. Ver [3]

\section{Análisis de la convergencia}

Se analizará si la sucesión de soluciones $\left(u_{h}\right)$ que se ha obtenido para cada valor de h, converge de alguna manera a la solución de (5) cuando $h \rightarrow 0$.

Teorema 2.9. Sea $\left(u_{h}\right)$ una familia de soluciones obtenida, entonces:

Se puede extraer una subsucesión $\left(u_{h^{\prime}}\right)$ tal que $\operatorname{lím}_{h^{\prime} \rightarrow 0}\left\|u_{h^{\prime}}-u\right\|_{H_{0}^{1}}=0$, donde u es solución del problema (5).

Esto se da para todas las posibles u que sean puntos de acumulación de $\left(u_{h}\right)$.

Demostración. Ver [4] 


\section{Cálculo de la solución aproximada}

Consideremos el problema de Burgers (5) y el subespacio aproximante $V_{0 h}$ dado en (6). La formulación variacional aproximada del problema es:

Hallar $u_{h} \in V_{0 h}$ tal que

$$
\alpha \int_{0}^{1} u_{h} v_{h} d x+\int_{0}^{1} u_{h} \frac{d u_{h}}{d x} v_{h} d x+\gamma \int_{0}^{1} \frac{d u_{h}}{d x} \frac{d v_{h}}{d x} d x=\int_{0}^{1} f_{h} v_{h} d x, \forall v_{h} \in V_{0 h}
$$

donde $u_{h}=\sum_{i=1}^{N} u_{i} w_{i} \quad$ y $\left\{w_{i}\right\}_{i=1}^{N}$ es una base de $V_{0 h}$. El problema se puede escribir como Hallar $u_{h} \in V_{0 h}$ tal que

$$
\alpha \int_{0}^{1} u_{h} w_{i} d x+\int_{0}^{1} u_{h} \frac{d u_{h}}{d x} w_{i} d x+\gamma \int_{0}^{1} \frac{d u_{h}}{d x} \frac{d w_{i}}{d x} d x=\int_{0}^{1} f_{h} w_{i} d x, i=1,2, \ldots, N
$$

que es un sistema no lineal con $\mathrm{N}$ ecuaciones en las incógnitas $u_{1}, u_{2}, \ldots, u_{N}$. Por tanto, la solución de (9) solo se realizará de forma aproximada por algún algoritmo iterativo.

\section{Matriz de precondicionamiento}

Sea $J: R^{N} \mapsto R$ derivable, considerar el problema de optimización.

Halla $x \in R^{N}$ tal que

$$
J(x) \leq J(y), \quad \forall y \in R^{N}
$$

Teorema 2.10. (Teorema de existencia) Sean $U \subset R^{N}$ cerrado y no vacío y $J: R^{N} \mapsto R$ una función continua y coerciva (si $U$ no está acotado). Entonces existe al menos un elemento u tal que

$$
J(u)=\inf _{v \in U} J(v)
$$

Si además $J$ es estrictamente convexo, la solución es única.

Demostración. Ver [3] , [4]

Teorema 2.11. (Condición necesaria y suficiente) Sean $V$ un espacio normado, $U \subset V$ convexo y $J: U \subset V \mapsto R$ una función convexa y derivable en $u \in U$. entonces

$$
J(u)=\inf _{v \in U} J(v) \Leftrightarrow J^{\prime}(u)(v-u) \geq 0, \quad \forall u \in U
$$

Demostración. Ver [4]

En las condiciones del teorema anterior, si $U=V=R^{N}$ la condición necesaria y suficiente es

$$
J^{\prime}(u)=0
$$

Ahora veamos el problema.

Hallar $x \in R^{N}$ tal que

$$
J^{\prime}(u)=0 \quad \text { con } \quad J^{\prime}(y)=\left[\begin{array}{c}
\frac{\partial J(y)}{\partial x_{1}} \\
\vdots \\
\frac{\partial J(y)}{\partial x_{N}}
\end{array}\right]
$$

Vamos a construir una sucesión $\left(x_{n}\right)$ que tienda a la solución $x$.

Consideremos una matriz $S \in M_{N \times N}$ denominada matriz precondicionamiento, tal que:

1. La matriz S permanece constante en todas las iteraciones.

2. La matriz S es simétrica y definida positiva. De esta manera se puede factorizar S usando el método de Choleski al principio del algoritmo, obteniendo asi un considerable ahorro de cálculo. 


\section{Elección de la matriz precondicionamiento}

Considerar la matriz S lo más sencillo posible. Una elección clásica de S es

$$
S=\left(\begin{array}{ccc}
a_{11} & \ldots & 0 \\
\vdots & \ddots & \vdots \\
0 & \ldots & a_{N N}
\end{array}\right) \quad \text { definida positiva }
$$

Caso particular. Consideremos el problema:

Hallar $x \in R^{N}$ tal que

$$
A x+\Psi(x)=b
$$

donde

1. $A \in M_{N \times N}$ simétrica y definida positiva

2. $R^{N} \rightarrow R^{N}$ definida por $\Psi(y)=\left\{\phi\left(y_{i}\right)\right\}_{i=1}^{N} \quad \forall y=\left\{y_{i}\right\}_{i=1}^{N} \in R^{N}$

3. $R \rightarrow R$ continua y creciente.

La relación $(\mathrm{P})$ es un sistema no lineal y monótono que admite una única solución.

Si $F(y)=A y+\Psi(y)-b$. Se prueba que $F(y)=J^{\prime}(y)$ donde

$$
J(y)=\frac{1}{2}(A y, y)-(b, y)+\sum_{j=1}^{N} \Phi\left(y_{i}\right)
$$

con $\Phi$ primitiva de $\phi$ que se anula en 0

$$
\Phi(t)=\int_{0}^{t} \phi(\xi) d \xi
$$

Sea el problema:

Hallar $x \in R^{N}$ tal que

$$
J(x) \leq J(y), \quad \forall y \in R^{N}
$$

Aplicando el teorema (3.8) se tiene la existencia y unicidad de solución de (M), pues

1. $U=V=R^{N}$

2. $J$ es un funcional estrictamente convexo y derivable.

Luego, tenemos la existencia y unicidad de solución de (P). Ademas por el teorema (3.9), (P) y (M) son equivalentes.

Si ademas $\phi$ es derivable, entonces $J \in C^{2}\left(R^{N}\right)$; en este caso la elección natural de la matriz precondicionamiento es $S=A$, si A es fácilmente invertible.

\section{Método de mínimos cuadrados}

El método de mínimos cuadrados, para mas detalles ver [5].

Sea $\mathrm{V}$ un espacio de Hilbert real, dotado de un producto escalar (., .), con \|. || la norma asociada. Para un operador (no lineal) $A: V \rightarrow V^{\prime}$, se desea resolver la ecuación:

Hallar $u \in V$ tal que

$$
A(u)=0
$$


Denotemos por $\langle.,$.$\rangle la dualidad V^{\prime} V$, y por $\|.\|_{*}$, la norma en el dual, es decir

$$
\|f\|_{*}=\sup _{v \in V \backslash\{0\}} \frac{|\langle f, v\rangle|}{\|v\|}
$$

Resolver el problema (11) es resolver el problema de optimización:

Hallar $v \in V$ tal que

$$
\|A(u)\|_{*} \leq\|A(v)\|_{*} \quad \forall v \in V
$$

y, claramente, toda solución de (11) lo es de (12). Recíprocamente, si (11) admite solución, entonces toda solución de (12) también lo es de (11).

En la mayoría de los casos la norma dual $\|\cdot\|_{*}$ no es fácil de manejar, por tal motivo se introduce el isomorfismo de dualidad entre $\mathrm{V}$ y $\mathrm{V}$ '.

Definición 2.12. Se llama isomorfismo de dualidad al operador $S: V \rightarrow V^{\prime}$ que cumple

$$
(v, w)=\langle S v, w\rangle \quad \forall v, w \in V
$$

Algunas propiedades del operador S

1. $\langle S v, w\rangle=\|v\|^{2} \quad \forall v \in V$

2. $\left\langle f, S^{-1} f\right\rangle=\|f\|_{*}^{2} \quad \forall f \in V^{\prime}$

3. $\|S v\|_{*}=\|v\| \quad y \quad\left\|S^{-1} f\right\|^{2}=\|f\|_{*}$.

Veamos ahora el problema, dado $v \in V$.

Hallar $y_{v} \in V$ tal que

$$
S y_{v}=A(v)
$$

Dado que $\|A(v)\|_{*}=\left\|S y_{v}\right\|_{*}=\left\|y_{v}\right\|$, si definimos

$$
J(v)=\frac{1}{2}\left\|y_{v}\right\|^{2}
$$

se puede plantear el problema (12) de forma equivalente como el problema de mínimos cuadrados siguiente

Hallar $v \in V$ tal que

$$
J(u) \leq J(v) \quad \forall v \in V
$$

y ahora J sólo hace referencia a la norma sobre V (y no a la de V').

\section{Método del gradiente conjugado}

Sea $\mathrm{V}$ un espacio de Hilbert y $J: V \rightarrow R$ diferenciable. Se quiere construir una sucesión minimizante para (14), para ello usaremos el método del gradiente conjugado. Ver [2], [5]. Una formulación general es la siguiente:

\section{INICIO}

1. Elegir $u_{0} \in V$ arbitrario.

2. $\left\{\begin{array}{c}\text { Hallar } d_{0} \in V \quad \text { tal que } \\ \left(d_{0}, v\right)=\left\langle J^{\prime}\left(u_{0}\right), v\right\rangle \quad \forall v \in V\end{array}\right.$ 
3. $w_{0}=d_{0}$

DESCENSO Dados $n \geq 0 \quad$ y $u_{n}, d_{n}, w_{n} \in V$

4. $\left\{\begin{array}{c}\text { Hallar } \rho_{0} \in R^{+} \text {tal que } \\ J\left(u_{n}-\rho_{n} w_{n}\right)=\inf _{\rho>0} J\left(u_{n}-\rho w_{n}\right)\end{array}\right.$

5. $u_{n+1}=u_{n}-\rho_{n} w_{n}$

\section{NUEVA DIRECCION DE DESCENSO}

6. $\left\{\begin{array}{c}\text { Hallar } d_{n+1} \in V \text { tal que } \\ \left(d_{n+1}, v\right)=\left\langle J^{\prime}\left(u_{n+1}\right), v\right\rangle \quad \forall v \in V\end{array}\right.$

7. Si $d_{n+1}=0$, parar; $u_{n+1}$ es solución.

8. $\quad \gamma_{n}=\left\{\begin{array}{c}0 \text { algoritmo del gradiente con paso optimo (AGPO) } \\ \frac{\left(d_{n+1}, d_{n+1}\right)}{\left\|d_{n}\right\|^{2}} \text { algoritmo del gradiente de Fletcher-Reeves (AGFR) } \\ \frac{\left(d_{n+1}-d_{n}, d_{n+1}\right)}{\left\|d_{n}\right\|^{2}} \text { algoritmo del gradiente de Polck-Ribiere (AGPR) }\end{array}\right.$

9. $w_{n+1}=d_{n+1}+\gamma_{n} w_{n}$. Volver a la etapa (4), con $n+1$ en lugar de $n$.

\section{Aplicación del (AGPR) a la ecuación de Burgers}

Consideremos el problema de Burgers cuya formulación variacional es Hallar $u \in H_{0}^{1}(0,1)$ tal que

$$
\alpha \int_{0}^{1} u v d x+\int_{0}^{1} u \frac{d u}{d x} v d x+\gamma \int_{0}^{1} \frac{d u}{d x} \frac{d v}{d x} d x=\int_{0}^{1} f v d x, \forall v \in H_{0}^{1}(0,1)
$$

es un problema del tipo (13) con

$$
\langle A(u), v\rangle=\alpha \int_{0}^{1} u v d x+\int_{0}^{1} u \frac{d u}{d x} v d x+\gamma \int_{0}^{1} \frac{d u}{d x} \frac{d v}{d x} d x-\int_{0}^{1} f v d x
$$

Consideremos un producto escalar en $H_{0}^{1}(0,1)$ tomando la parte bilineal del problema, es decir

$$
(u, v)_{H_{0}^{1}}=\alpha \int_{0}^{1} u v d x+\gamma \int_{0}^{1} \frac{d u}{d x} \frac{d v}{d x} d x
$$

Definimos el isomorfismo de dualidad

$$
\langle S(v), w\rangle=\alpha \int_{0}^{1} v w d x+\gamma \int_{0}^{1} \frac{d v}{d x} \frac{d w}{d x} d x
$$

por tanto

$$
J(v)=\frac{\alpha}{2} \int_{0}^{1} y_{v}^{2} d x+\frac{\gamma}{2} \int_{0}^{1}\left(\frac{d y_{v}}{d x}\right)^{2} d x
$$

donde, a cada $v$ se le asocia $y_{v}$ solución de (15), es decir

Hallar $y_{v} \in H_{0}^{1}(0,1)$ tal que

$$
\begin{gathered}
\alpha \int_{0}^{1} y_{v} w d x+\gamma \int_{0}^{1} \frac{d y_{v}}{d x} \frac{d w}{d x} d x=\alpha \int_{0}^{1} v w d x+\int_{0}^{1} v \frac{d v}{d x} w d x+\gamma \int_{0}^{1} \frac{d v}{d x} \frac{d w}{d x} d x- \\
\int_{0}^{1} f w d x, \forall w \in H_{0}^{1}(0,1)
\end{gathered}
$$


Además

$$
\left\langle J^{\prime}(v), w\right\rangle=\alpha \int_{0}^{1} y_{v} w d x+\gamma \int_{0}^{1} \frac{d y_{v}}{d x} \frac{d w}{d x} d x+\int_{0}^{1} y_{v} \frac{d y_{v}}{d x} w d x+\gamma \int_{0}^{1} y_{v} v \frac{d w}{d x} d x
$$

Entonces el algoritmo queda como

\section{INICIO}

1. Elegir $u_{0} \in H_{0}^{1}(0,1)$ arbitrario

2.1

$$
\left\{\begin{array}{c}
\text { Hallar } y_{0} \in H_{0}^{1}(0,1) \text { tal que } \\
\alpha \int_{0}^{1} y_{0} w d x+\gamma \int_{0}^{1} \frac{d y_{0}}{d x} \frac{d w}{d x} d x=\alpha \int_{0}^{1} u_{0} w d x+\int_{0}^{1} u_{0} \frac{d u_{0}}{d x} w d x \\
+\gamma \int_{0}^{1} \frac{d u_{0}}{d x} \frac{d w}{d x} d x-\int_{0}^{1} f w d x, \quad \forall w \in H_{0}^{1}(0,1)
\end{array}\right.
$$

2.2

$$
\left\{\begin{array}{c}
\text { Hallar } g_{0} \in H_{0}^{1}(0,1) \text { tal que } \\
\alpha \int_{0}^{1} g_{0} v d x+\gamma \int_{0}^{1} \frac{d g_{0}}{d x} \frac{d v}{d x} d x=\alpha \int_{0}^{1} y_{0} v d x+\gamma \int_{0}^{1} \frac{d y_{0}}{d x} \frac{d v}{d x} d x \\
+\int_{0}^{1} y_{0} \frac{d u_{0}}{d x} v d x+\int_{0}^{1} y_{0} u_{0} \frac{d v}{d x} d x, \quad \forall v \in H_{0}^{1}(0,1)
\end{array}\right.
$$

3. $w_{0}=g_{0}$

DESCENSO Dados $n \geq 0 \quad$ y $u_{n}, g_{n}, w_{n} \in H_{0}^{1}(0,1), \quad w_{n} \neq 0$

4.

$$
\left\{\begin{array}{c}
\text { Hallar } \rho_{0} \in R^{+} \text {tal que } \\
J\left(u_{n}-\rho_{n} w_{n}\right)=\inf _{\rho>0} J\left(u_{n}-\rho w_{n}\right)
\end{array}\right.
$$

5. $\quad u_{n+1}=u_{n}-\rho_{n} w_{n}$

\section{NUEVA DIRECCION DE DESCENSO}

6.1

$$
\left\{\begin{array}{c}
\text { Hallar } y_{n+1} \in H_{0}^{1}(0,1) \quad \text { tal que } \\
\alpha \int_{0}^{1} y_{n+1} w d x+\gamma \int_{0}^{1} \frac{d y_{n+1}}{d x} \frac{d w}{d x} d x=\alpha \int_{0}^{1} u_{n+1} w d x+\int_{0}^{1} u_{n+1} \frac{d u_{n+1}}{d x} w d x \\
+\gamma \int_{0}^{1} \frac{d u_{n+1}}{d x} \frac{d w}{d x} d x-\int_{0}^{1} f w d x, \quad \forall w \in H_{0}^{1}(0,1)
\end{array}\right.
$$

6.2

$$
\left\{\begin{array}{c}
\text { Hallar } g_{n+1} \in H_{0}^{1}(0,1) \quad \text { tal que } \\
\alpha \int_{0}^{1} g_{n+1} v d x+\gamma \int_{0}^{1} \frac{d g_{n+1}}{d x} \frac{d v}{d x} d x=\alpha \int_{0}^{1} y_{n+1} v d x+\gamma \int_{0}^{1} \frac{d y_{n+1}}{d x} \frac{d v}{d x} d x \\
+\int_{0}^{1} y_{n+1} \frac{d u_{n+1}}{d x} v d x+\int_{0}^{1} y_{n+1} u_{n+1} \frac{d v}{d x} d x, \quad \forall v \in H_{0}^{1}(0,1)
\end{array}\right.
$$


Si $g_{n+1}=0$, parar; $u_{n+1}$ es solución

7 .

$$
\gamma_{n}=\frac{\left(g_{n+1}-g_{n}, g_{n+1}\right)_{H_{0}^{1}}}{\left\|g_{n}\right\|_{H_{0}^{1}}^{2}}
$$

8.

$$
w_{n+1}=g_{n+1}+\gamma_{n} w_{n}
$$

Volver a la etapa (4), con $n+1$ en lugar de $n$. Ver [2], [5].

\section{Conclusión}

En este trabajo se estudia la ecuación viscosa de Burgers, esta ecuación es obtenida a partir de la ecuación de Euler agregando el término de "viscosidad artificial", y así no genere mayores dificultades al considerar el esquema de diferencias finitas. Esta misma técnica se puede aplicar al problema de Navier-Stokes que es un problema no lineal y es la que representa mejor a la ecuación de la dinámica de fluidos. 


\section{Referencias Bibliográficas}

[1] Amster, P. (2009). Métodos Topológicos en el Análisis no Lineal. IMPA. Rio de Janeiro, Brasil.

[2] Bristeau, M. O., Glowinski, R. (1987). Numerical methods for the Navier-Stokes equations. Computer Physics Reports Vol 6: 73-187.

[3] Conway, J. B. (1990). A course in Functional Analysis. Springer. New York, USA.

[4] Lions, J. L. (1969). Quelques Methodes de Resolution des Problemes aux Limites. Dunod. Paris, Francia.

[5] Quarteroni A., Sacco R., Salvi F. (2000). Numerical Mathematics. Springer-Verlag. 